\title{
HIGHLY SENSITIVE DETECTION OF LEAD IONS IN WATER USING ETCHED FBG
}

\author{
V.K.Kulkarni ${ }^{1}$,A.S.Lalasangi ${ }^{2}$, S.B.Patil ${ }^{3}$, S. R. Mannopantar ${ }^{4}$, M. S. Jadhav ${ }^{5}$ \& U.S.Raikar ${ }^{6}$ \\ ${ }^{1}$ Department Of Physics, Angadi Institute Of Technology \& Management, Belagavi, (Karnataka), \\ India. \\ 2. Department Of Physics, Smt. I. S. Yadawad Government First Grade College Ramdurg, \\ (Karnataka), India \\ ${ }^{3}$ Department Of Physics, Government First Grade College, Ranebennur (Karnataka) India. \\ 4. Department Of Physics, Government First Grade College, Harihar, (Karnataka) India \\ ${ }^{5}$ Department Of Physics, J. S. S. Arts, Science And Commerce College, Gokak (Karnataka) India. \\ ${ }^{6}$ Department Of Physics, Karnatak University, Dharwad (Karnataka) India.
}

\begin{abstract}
Lead is a soft, ductile, malleable and naturally present in earth's crust. Lead is highly toxic element affects living organism even at lower concentration. Lead is used in many industries and its disposable is very difficult. Therefore, it may lead to various environmental hazardous problems. World Health Organization (WHO) and other organisation specify the presence of lead in drinking water not greater than $20 \mathrm{ppb}$. In the present work, a simple, affordable and precise way to detect the concentration of lead in water using Fibre Bragg gratings (FBG) is presented. FBG are formed using Phase mask technique and the cladding part over the grating region is etched with $40 \%$ Hydrofluoric acid (HF) solution. The sensor so developed is sensitive enough to find the presence of lead in water in the range 5-25 ppb.
\end{abstract}

Keywords: Fibre Bragg grating, Etching, Lead, sensor, ppb.

\section{INTRODUCTION}

Lead poisoning seems to be one of the major causes for critical health concern across world. It has been treated as one of the most harmful metal concern with public health environment[1,2]. Pollution caused due to heavy metals like mercury, arsenic, lead, copper, fluoride, zinc, chromium and many more which leads to major health issues in humans and animals[3,4]. It enters in the living system through ingestion of contamination in water and food materials [5-9]. As lead is not present in drinking water unless it is being contaminated during the transportation through metal pipes, PVC pipes and even through corrosion of plumbing material and solder, which may contain lead. The quantity of lead in water may even depends on the ages of pipes, duration of water present in such pipes, minerals present in water, temperature and $\mathrm{pH}$ level of water[10,11].

$$
\text { Techniques reported various }
$$

measurements in connection with identifying the presence of lead in water. The most common method is utilising the atomic absorption spectrometry technique and with this method most accurate measurement can be recorded[12,13]. The other methods reported are through Fluorescent technique and using optical fibres $[5,14,15]$.

\section{THEORY AND WORKING PRINCIPLE OF FBG SENSOR}

Bragg gratings have great potential to operate in wavelength of $1350 / 1550 \mathrm{~nm}$ in optical communication system and such gratings are fabricated by exposing them to UV source. FBG are a sort of periodic variation in refractive index of core in a single mode photosensitive optical fibre [16].

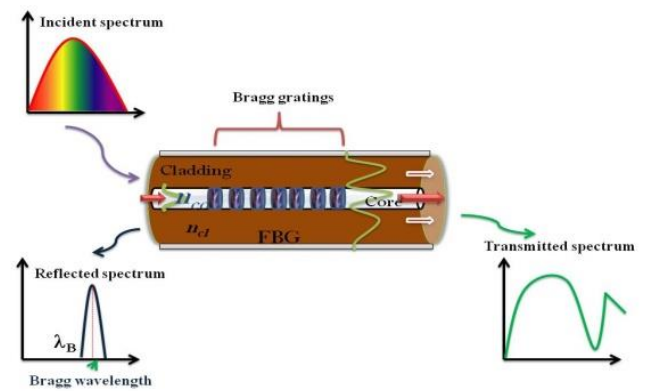

Fig.1. The transmitted / reflected spectra of fiber grating.

The grating fabrication is carried out by the phase mask technique[7]. When a broad band source is connected to the one end of the FBG, we observe a narrow band of spectrum is reflected 


\section{International Journal of Engineering Applied Sciences and Technology, 2021 \\ Vol. 6, Issue 3, ISSN No. 2455-2143, Pages 122-126 \\ Published Online July 2021 in IJEAST (http://www.ijeast.com)}

back which is shown in Fig.1. This reflected spectra seems to very sensitive towards external perturbation $[17,18]$. Such FBG sensors are highly sensitive lower concentration mainly ppb level in liquid detection. This FBG is used as a chemical sensor to find out the contaminants in drinking water.

The present FBG is designed for chemical sensing. The cladding section of the grating region is to be etched, so that core modes interaction takes place directly with the surrounding environment directly[6,13,14]. This enhances the matching of effective refractive index of waveguide mode with surrounding region. When such etched region of grating is immersed in external liquid, leads to shift in Bragg wavelength $\left(\boldsymbol{\delta} \lambda_{\boldsymbol{B}}\right)$ given by the following relation[7].

$$
\delta \lambda_{B}=2 \Lambda \delta n \delta \eta_{p}
$$

Where, $\boldsymbol{\Lambda}$ is grating period, $\mathbf{n}$ corresponds to variation of refractive index of cladding and surrounding region and $\boldsymbol{\delta} \boldsymbol{\eta}_{\mathbf{p}}$ corresponds to change in fraction of unperturbed mode power, which enters in to the etched region.

\section{EXPERIMENTAL PROCEDURE}

The grating region is to be etched in such a way that complete cladding region has to be taken out. Initially broad band source is launched though $3 \mathrm{~dB}$ coupler in to the FBG and optical spectrum analyser (OSA). The wet chemical etching of the fiber cladding is carried out using $40 \% \mathrm{HF}$ placed in a Teflon container. The etching process is very closely monitored through the OSA. Around 55 minutes, a sudden shift in wavelength was observed and grating region was removed from HF solution. This clearly indicates the etching of cladding region completely. This etched grating region is now washed with distilled water and followed by isopropyl alcohol solution. Now this etched FBG is set ready for further experimental procedure. Fig.2. shows the variation of spectra before after etching process.

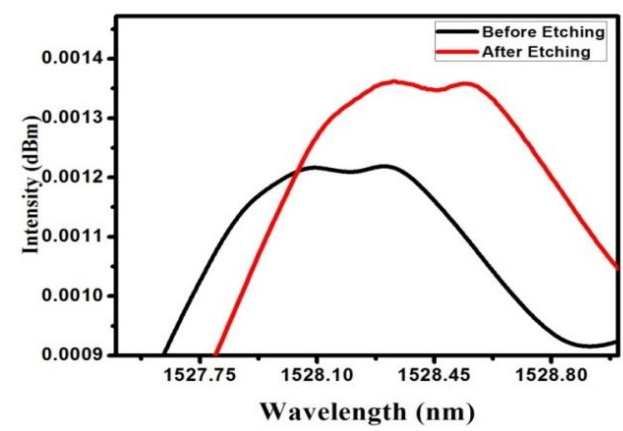

Fig.2. Etching process with FBG

Etching of FBG at sensing region has been carried out in order to make more sensitive for surrounding environment. In present report, designed etched FBG is used to detect and determine the lead contaminant in water at $\mathrm{ppb}$ level.

The test solution for the Lead is prepared by dissolving Lead nitrate salt in distilled water. The stock solution is prepared and concentration varying from $5 \mathrm{ppb}$ to $25 \mathrm{ppb}$ is prepared by further diluting the stock solution. For each $10 \mathrm{ml}$ of test solution $0.4 \mathrm{ml}$ reagents namely Ethanol, Ammonia, Pottassium Cyanide, hydroxylammonium chloride and Dithizone are added in a sequence mentioned. Now this test solution is mixed thoroughly. In the presence of Pottassium Cyanide, lead (II) ions interacts with Dithizone to form lead Dithizonate to form a pink colour solution.

The experimental setup to detect lead ions in water at room temperature is shown in Fig.3. The Broad Band Source of $17 \mathrm{~mW}$ (JDSUNIPHASE) of wavelength $1530-1600 \mathrm{~nm}$ is launched through $3 \mathrm{~dB}$ coupler $(2 \times 2)$ in to the FBG. One of its output ends is connected to the FBG and the other end is free. The FBG is immersed in test solution containing different concentration of lead solution. The reflected spectrum is observed through Optical Spectrum Analyzer (OSA) [ANRITSU] connected through one of the free input end of $3 \mathrm{~dB}$ coupler.

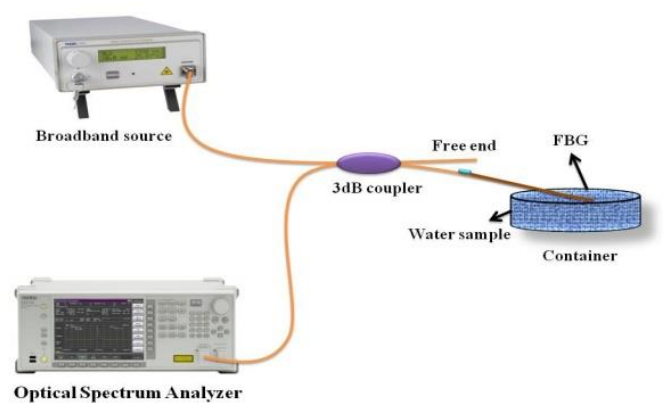

Fig.3. Experimental setup for the detection of lead in water

Standard test solutions of lead varying from $5 \mathrm{ppb}$ to $25 \mathrm{ppb}$ are kept ready for experimental work. Initially it is recorded with lower concentration of $5 \mathrm{ppb}$ and this test solution is transferred in to groove made-up Teflon container in which the etched grating portion is placed. When broad band source is launched in to FBG through $3 \mathrm{~dB}$ coupler, the reflected spectra from FBG is recorded through OSA. Further the process is continued for other higher concentration of lead in an increasing 
order. During each reading the Teflon container is cleaned thoroughly with isopropyl alcohol and distilled water to avoid contamination. When test solution is placed in Teflon container the effective refractive index of FBG core mode changes, and leads to shift in Bragg wavelength $\left(\lambda_{\mathbf{B}}\right)$. It shows that as the concentration of the solution varies there is a change in Bragg wavelength.

\section{RESULTS AND DISCUSSION}

The detection of lead in water using FBG is carried out at room temperature. The readings were taken from lower concentration of $5 \mathrm{ppb}$ to $25 \mathrm{ppb}$ and corresponding changes of wavelength, intensity and concentration were tabulated. Firstly the variation of intensity with concentration of lead is shown in Fig 4 (a) \& (b).
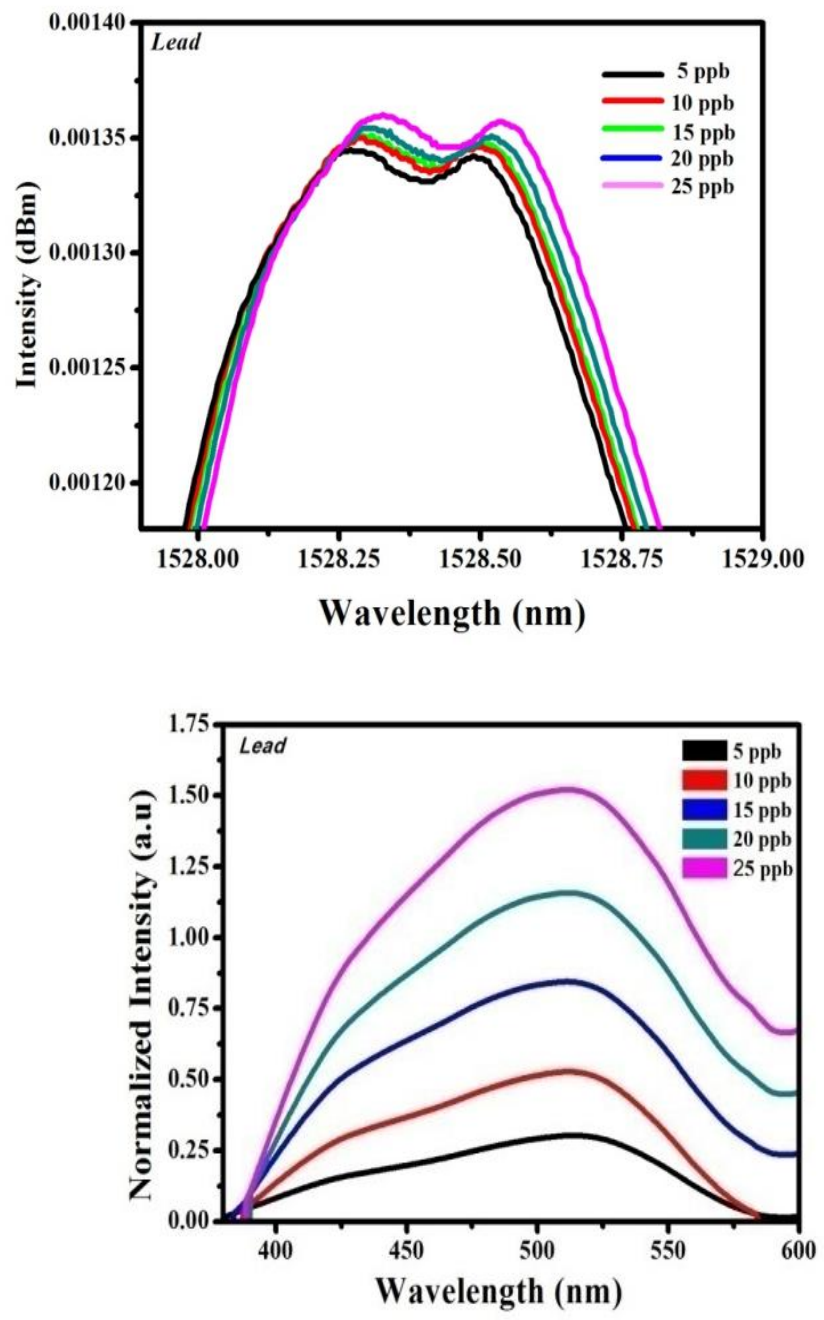

Fig.4. (a) intensity Vs wavelength spectrum (b) Normalized spectrum for various concentrations of samples.
It is clearly evident from the graph that as lead concentration goes on increasing the intensity and wavelength shifts are clearly distinguishable. When intensity versus wavelength variation is plotted the variation of $2.4617 \mathrm{dBm} / \mathrm{nm}$ is observed as shown in Fig.5.

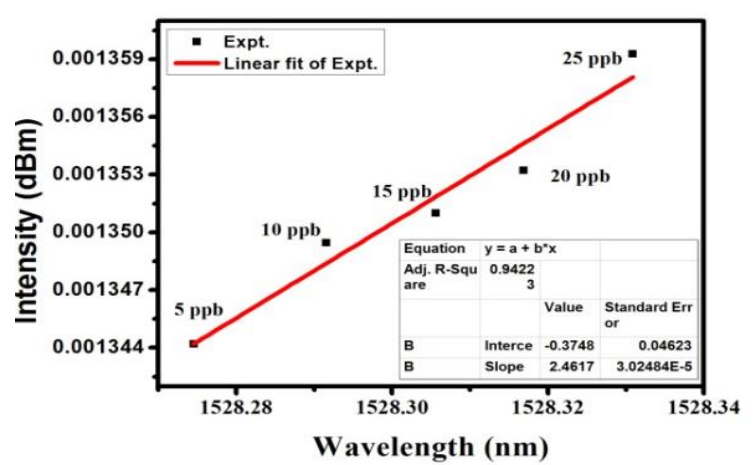

Fig.5. Linear fit of intensity Vs Wavelength for lead detection

The variation of wavelength against concentration of lead is shown in Fig.6. and the high sensitivity was observed of $0.00276 \mathrm{~nm} / \mathrm{ppb}$. As FBG based sensor is wavelength dependent and is quite sensitive one. Thus, the sensor shows a very high sensitivity in detection of presence of lead in water using FBG.

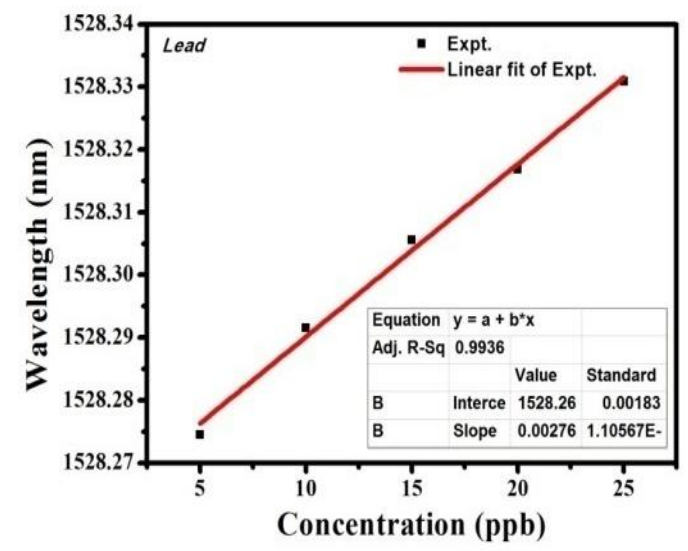

Fig.6. Linear fit of wavelength Vs concentration for lead detection

Similarly the graph of Intensity versus concentration was plotted as shown in Fig. 7 and the sensitivity is $6.7841 \mathrm{dBm} / \mathrm{ppb}$. Thus our sensor so developed is sensitive enough in detecting the presence of lead in water within the permissible standard level given by various authorities[19]. 


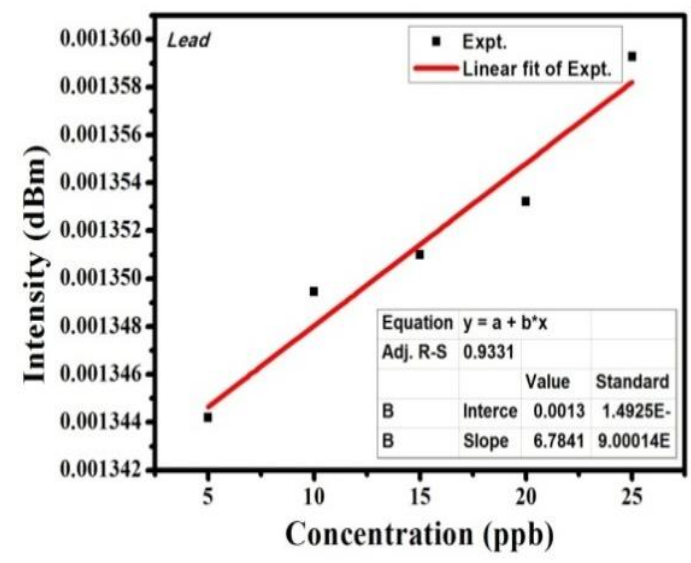

Fig.7. Linear fit of intensity Vs concentration for lead detection

It reveals that as increase in the sample concentration Bragg shift occurs with increase in intensity of the signals and sensitivity 6.7841 $\mathrm{dBm} / \mathrm{ppb}$ at lower concentration. It confirms that designed sensor is highly sensitive at lower concentration in detecting the chemical species.

\section{CONCLUSION}

The variation shift in Bragg wavelength is observed as the concentration goes on increasing. It shows a red shift in wavelength as the intensity decreases. The high sensitivity observed is 0.00276 $\mathrm{nm} / \mathrm{ppb}$. The above all results reveal that the FBG chemical sensor is one of the sensitive and desirable components in detection of lead in water. In FBG Bragg wavelength is the characteristic parameter involved in building such sensors. The measurement techniques are not affected with perturbations such as variation in input power and thus capable enough in absolute measurement of the measurand. The detection limits are far better than other methods reported. Thus FBG chemical sensor proves to be a potential tool in measuring the heavy metal like lead (II) present in water. Our detection level is very high within the range of limitations set by WHO and other agencies. The sensor so developed is quite affordable, simple in design and reproducible.

\section{ACKNOWLEDGMENT}

The present work is supported by the VGST Karnataka Science and Technology Promotion Society (KSTePS) Department of IT, BT and S\&T Government of Karnataka, Bengalooru., INDIA.

\section{REFERENCES}

[1] S. S. Chong, A. R. A. Aziz, and S. W.
Harun, Fibre Optic Sensors for Selected Wastewater Characteristics, 13(7), 2013, pp. 86408668 .

[2] L. Wong, R. Deo, S. Rathnayaka, B. Shannon, and C. Zhang, Leak Detection in Water Pipes Using Submersible Optical Optic-Based Pressure Sensor, 18(12), 2018, pp. 4192 (1-17) .

[3] T. M. Libish et al.,Optik Detection and analysis of paraffin oil adulteration in coconut oil using fiber optic long period grating sensor, Optik International Journal for Light and Electron Optics, 122 (21), 2011, pp. 1939-1942,

[4] U. S. Raikar et al,. Chemical Cd concentration sensor based on fiber grating technology, Sensors \& Actuators: B. Chemical, 161(1), 2012, pp. 818-823,.

[5] A. M. Valadez, C. A. Lana, S. I. Tu, M. T. Morgan, and A. K. Bhunia, "Evanescent wave fiber optic biosensor for Salmonella detection in food," Sensors, 9, 2009, pp. 5810-5824.

[6] L. S. Laxmeshwar, M. S. Jadhav, J. F. Akki, P. Raikar, and U. S. Raikar,Elemental analysis of wastewater effluent using highly sensitive fiber Bragg grating sensor, Optics and Laser Technology, 105, 2018,pp. 45-51.

[7] L. S. Laxmeshwar et al., "Highly sensitive fiber grating chemical sensors: An effective alternative to atomic absorption spectroscopy," Optics \& Laser Technology, 91, 2017,pp. 27-31.

[8] L. S. Laxmeshwar et al., Chemical Quantification of chloride and iron in sugar factory effluent using long period fiber grating chemical sensor, Sensors \& Actuators: B. Chemical, vol. 258, 2018, pp. 850-856.

[9] M. S. Jadhav, L. S. Laxmeshwar, J. F. Akki, P. U. Raikar, and J. Kumar, "Optical Fiber Technology Fluoride contamination sensor based on optical fi ber grating technology," Optical Fiber Technology, vol. 38, no. September, 2017, pp. 136141.

[10] A review of permissible limits of drinking water $M$ Kumar and A. Puri, A review of permissible limits of drinking water, Indian $J$ Occup Environ Med. 16(1),2012,pp. 40-44.

[11] M. J.Brown and S.Margolis, Lead in Drinking Water and Human Blood Lead Levels in the United States. Supplements. CDC (Morbidity and Mortality Weekly Report MMWR) 61(04),2012, 1-9. 
[12] Jyoti F. Akki, Anandkumar S. Lalasangi, Prasad U. Raikar, T. Srinivas, Lata S. Laxmeshwar, and U. S. Raikar, Core-cladding mode resonances of long period fiber grating in concentration sensor, IOSR Journal of Applied Physics (IOSR-JAP), 4(3), 2013, pp. 41-46,.

[13] S. D. Richardson, Disinfection byproducts and other emerging contaminants in drinking water, TrAC - Trends in Analytical Chemistry, 22(10), 2003, pp. 666-684.

[14] C. A. Villarruel, D. D. Dominguez, and A. Dandridge, "Evanescent Wave Fiber Optic Chemical Sensor," Proceddings of SPIE, 798, 1987,pp. 225-229.

[15] Y. J. Rao, "Recent progress in applications of in-fibre Bragg grating sensors," Optics and Lasers in Engineering,31(4),1999, pp. 297-324.

[16] B. Lee, Review of the present status of optical fiber sensors, Optical Fiber Technology, 9(2), 2003,pp. 57-79..
[17] M. Latino, R. Montanini, N. Donato, and G. Neri, Ethanol sensing properties of PMMAcoated fiber Bragg grating, Procedia Engineering, 47, 2012, pp. 1263-1266,.

[18] M. B. Rao, M. R. Bhat, K. V. Madhav, and S. Asokan, "Structural Health Monitoring ( SHM ) Using Strain Gauges, PVDF Film and Fiber Bragg Grating ( FBG ) Sensors: A Comparative Study,"2006. https://www.researchgate.net /publication/239821755

[19] J. Fawell and M. J. Nieuwenhuijsen, Contaminants in drinking water,. DOI: $10.1093 / \mathrm{bmb} / \mathrm{ldg} 027$ 